\title{
Regular black holes with exotic topologies
}

\author{
Gustavo Melgarejo* \\ Departamento de Física, Universidad de Los Andes, A. A. 4976 , Bogotá, Colombia \\ Ernesto Contreras ${ }^{\dagger}$ \\ Departamento de Física, Colegio de Ciencias e Ingeriería, \\ Universidad San Francisco de Quito, Quito, Ecuador \\ Pedro Bargueño \\ Departamento de Física Aplicada, Universidad de Alicante, \\ Campus de San Vicente del Raspeig, E-03690 Alicante, Spain
}

(Dated: February 18, 2021)

\begin{abstract}
In this work we show that spherically symmetric regular black holes with topologies other than $S^{3}$ de Sitter cores can not be excluded from Borde's theorem. In particular, we prove that spacetime connectivity leads to an $S^{3}$ core by discarding other allowed topologies, such as the orientable and no orientable $S^{2}$ bundles over $S^{1}$, for achronal slices. Finally, some conjectures regarding physical quantification of topology change in these spacetimes are discussed.
\end{abstract}

\section{INTRODUCTION}

The 1965 Penrose theorem ${ }^{1}$ proved that generic singularities are unavoidable, provided some physical conditions are satisfied. Therefore, as physical laws are undefined in presence of these singularities, in order to guarantee the existence of the former, the latter have to be substituted by some other object in an improved theory, usually taken to be some approximation to a quantum theory of gravity. However, the difficulty of formulating a complete and consistent theory encompassing General Relativity within the quantum realm has give place to a line of work which deals with classical (or semiclassical) black holes with regular (non singular) properties.

The first solution for a regular black hole was proposed by Bardeen in $1968^{2}$, who realized the idea of Sakharov ${ }^{3}$ and Gliner ${ }^{4}$, proposing that singularities could be substituted by an inflationary equation of state (i. e. a de Sitter core). Since then, although there have been a great development within the field, most of regular solutions which are continuous throughout the whole spacetime were constructed following Bardeen's proposal ${ }^{38}$. Without trying to be exhaustive, we would like to mention some works along this line. In particular, Dymnikova ${ }^{5-8}$ found a regular black hole solution whose interior corresponds to an anisotropic fluid obeying a de Sitter equation of state, $p=-\rho$, that was asymptotically Schwarzschild. Other regular black hole solutions with a de Sitter core were found by employing nonlinear electrodynamics as the source $\mathrm{e}^{9-12}$. In addition, regular black holes also appeared in different extended theories of gravity ${ }^{13-15}$. Interestingly, regular black hole solutions with a Minkowski core have been recently pointed out ${ }^{16}$, allowing thus to explore new possibilities.

From a global point of view, there have been mainly two lines of attack to the problem. The first one was proposed by Borde during the 90s, who showed how a reversed Penrose theorem ${ }^{17}$ allowed, at the price of sacrifying global hiperbolicity, to explain the existence of a family of regular solutions with a de Sitter core by using global methods. The second one has been very recently developed by Carballo-Rubio, Di Filippo, Liberati and Visser ${ }^{18,19}$, who have shown a complete classification of spherically symmetric and geodesically complete black holes but this time by invoking global hiperbolicity for the whole spacetime. Specifically, they have clarified that most of regular black hole solutions do not evade Penrose's theorem by avoiding the formation of focusing points, as proposed in Refs. ${ }^{18,19}$, but rather by introducing a Cauchy horizon before this focusing point is reached by outgoing geodesics, in accordance with Borde's theorem.

In this article we take an intermediate step based on Borde's theorem to show that regular black hole solutions that are not asymptotically de Sitter but still maintain a Reissner-Nordström-like causal structure can be constructed. Specifically, the manuscript is organized as follows. Section II summarizes Borde's theorem, which relates topological changes and regular black holes via global techniques. In order to facilitate the reading, the main definitions and notations are established in this section. In section III we introduce the relevant Carter-Penrose diagrams for spherically symmetric, regular black holes with two horizons, and we provide the reader with a simplified proof of the necessity of a de Sitter core in the inner region. After regular black holes with exotic (orientable and no-orientable) topologies are constructed from lightlike compactifications of their CarterPenrose diagrams in Section IV, we conclude in Section $\mathrm{V}$ by conjecturing some relations between topological changes, physical quantities defined on the relevant slices and transitions between Petrov types. Finally, Section VI present a brief summary of our findings. 


\section{BORDE'S THEOREM}

Theorem. Suppose that there is a spacetime $\mathcal{M}$, that

1. $\mathcal{M}$ contains an eventually future-trapped surface $\mathcal{T}$.

2. The Ricci tensor, $R_{\mu \nu}$, obeys $R_{\mu \nu} n^{\mu} n^{\nu} \geq 0$ for all null vectors $n^{\mu} \in \mathcal{M}$.

3. $\mathcal{M}$ is null-geodesically complete to the future.

4. $\mathcal{M}$ is future causally simple, i.e., $E^{+}(X)=\dot{I}^{+}(X)$, where $X$ is any achronal compact subset of $\mathcal{M}$.

Then there is a compact slice to the causal future of $\mathcal{T}^{17}$.

The main ingredients of this theorem, which are crucial for a correct understanding of it, are an (eventually) trapped surface and an achronal hypersurface defined on a future-causally-simple spacetime. Let us briefly review their definitions.

A trapped surface is a two-surface in which both outgoing and ingoing null geodesics perpendicular to this surface are convergent, i.e., these null geodesics have negative divergence on this surface ${ }^{20}$. In addition, for a eventually future-trapped surface only is required that the divergences are negative somewhere in the future of the surface along each geodesic ${ }^{17}$. A subset $S \subset \mathcal{M}$ of an arbitrary spacetime $\mathcal{M}$ is said to be achronal if there does not exist a pair $p, q \in S$ such that it can be connected by causal curves ${ }^{21}$. A spacetime $\mathcal{M}$ is said future causally simple if $E^{+}(X)=\dot{I}^{+}(X)$, where $X$ is some compact achronal subset of $\mathcal{M} . E^{+}(X)$ is the future horismo of $X$, which is defined by $E^{+}(X)=J^{+}(X)-I^{+}(X)$, where $J^{+}(X)$ is the causal future of $X, I^{+}(X)$ is the chronological future of $X$, and $\dot{I}^{+}(X)$ is the boundary of $I^{+}(X)$. Finally, we must remember that a slice $\Gamma$ is an edgeless, achronal hypersurface; i. e. for every point $p \in \Gamma$ there is no timelike curve that can reach points $u \in I^{-}(p)$ and $v \in I^{+}(p)$.

\section{REGULAR BLACK HOLES WITH A DE SITTER CORE WITHIN SPHERICAL SYMMETRY}

As commented in the introduction, we are interested in spacetimes possessing two horizons, namely, an event horizon and a Cauchy horizon. The global structure of these spacetimes is similar to the maximal extension of the Reissner-Nordström spacetime, as Fig. (1) shows.

One possibility of obtaining a regular black hole spacetime is by identifying the regions corresponding to $r=0$ in the diagram of Fig. (1). When making the identification, the diagram of Fig. (2) is obtained, the shaded area representing those spacetime points that can be reached by time-like curves starting from $p$. In this case the spacetime is future causally simple since $E^{+}(p)=\dot{I}^{+}(p)$.

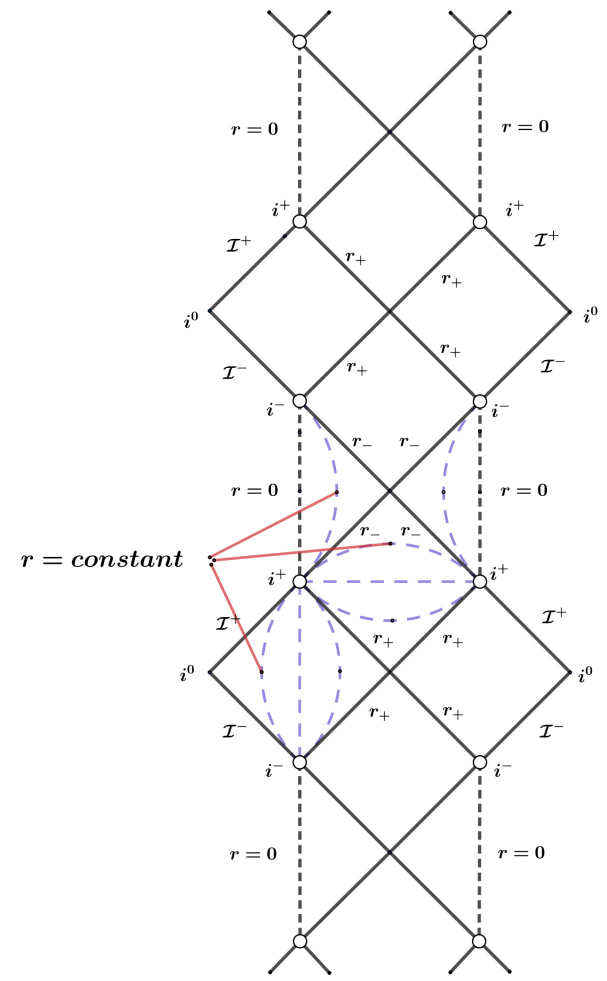

FIG. 1. Carter-Penrose diagram for a Reissner-Nordströmlike black hole.

Moreover, it is future null geodesically complete, as can be easily shown by inspection of Fig. (2).

Finally, let us impose that the null energy condition is fulfilled. Then, all the requirements of Borde's theorem are satisfied and, therefore, there must be some compact slice in the future of the trapped surface $p$.

Interestingly, this spacelike identification makes the topology of the slices in the internal region of the regular black hole to be $S^{3}$. In order to show it, let us introduce a new coordinate, which we denote by $\chi$, such that the rays of light that leave the point $p$ can move along this coordinate which wraps around the spacetime.

When we move through $\chi$, the temporal coordinate (in Schwarzschild coordinates) remains fixed, but the radial coordinate varies periodically. In addition, let us remember that each point in these diagrams represent a 2 -sphere. Therefore, in this region we can define a line element by

$$
d s^{2}=\alpha d \chi^{2}+r^{2}(\chi) d \Omega^{2}
$$

where $\alpha$ is a constant. Even more, by noting that $r(\chi)$ varies from $r=0$ to a maximum value at $r=r_{-}$to then decrease and get back to $r=0$, we can write

$$
r(\chi)=r_{-} \sin \chi, \quad 0 \leq \chi<2 \pi .
$$

Then, the line element in the inner region can be writ- 


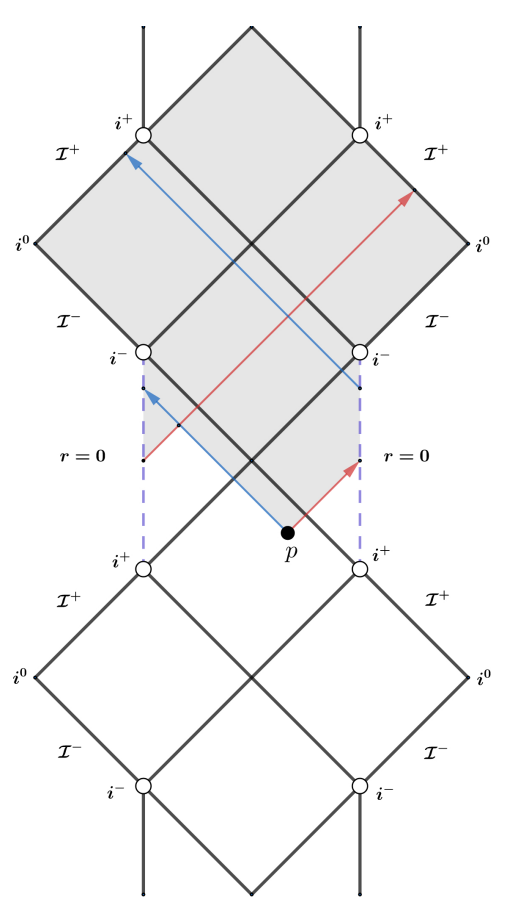

FIG. 2. Carter-Penrose diagram for space-like regularization.

ten as

$$
d s^{2}=r_{-}^{2} d \chi^{2}+r_{-}^{2} \sin ^{2} \chi d \Omega^{2},
$$

which clearly corresponds to that of a 3 -sphere, which is the topology of the spacelike sections of the de Sitter spacetime, written in canonical coordinates.

Finally, let us note that that there is a change in the topology of spacelike slices, which vary from $\mathbb{R} \times S^{2}$ in regions away from the core of the regular black hole to $S^{3}$ in the inner region of it.

At this point, several comments are in order: first, most solutions for regular black holes have the behavior described above, being known as Bardeen-like solutions ${ }^{6,8,22,23}$; second, these solutions tend to a de Sitter one as we approach $r=0$. This can be shown, for example, by looking at the spacelike diagram enclosed by the two $r=0$ regions of Fig. (2), which corresponds to that of a de Sitter spacetime or using the previous argument where a $S^{3}$ topology for the inner spacelike hypersurface is obtained; third, due to the de Sitter behavior, the null energy condition is saturated at $r=0$ $\left(R_{\mu \nu} n^{\mu} n^{\nu}=0\right.$ for all null vectors $n^{\mu}$ when $\left.r=0\right)$. And four, let us notice that the spacelike slices can also be identified for values of $r$ other that $r=0$, which open us the possibility of building an infinite family of solutions with a de Sitter center. In this last situation, in contrast with identifying the $r=0$ regions of the diagram, the range of variation of the $\chi$-coordinate is not restricted between 0 and $2 \pi$ and, therefore, the corresponding spacetime is not simply connected. This situation would correspond, for example, to the identifica-

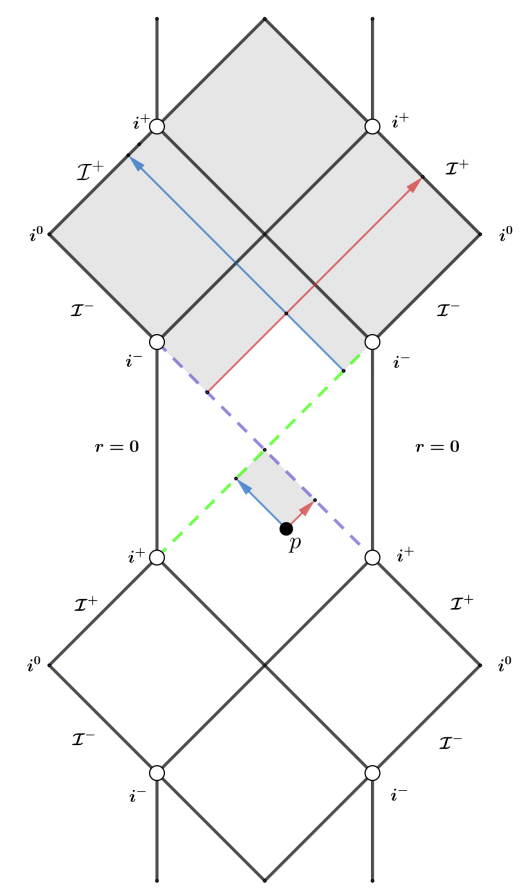

FIG. 3. Carter-Penrose diagram for null identification along the Cauchy horizon.

tion of the two vertical dashed curved lines lying between $r=0$ in Fig. (1).

\section{REGULAR BLACK HOLE SOLUTIONS WITH NON DE SITTER ASYMPTOTICS}

In the previous section we have shown that one can easily construct regular black hole solutions with a de Sitter core, which is equivalent to imposing $R_{\mu \nu} n^{\mu} n^{\nu}=0$ at $r=0$. Let us now consider the cases in which the null energy condition is satisfied but not saturated. Then, if the rest of the hypothesis regarding Borde's theorem are satisfied, a regular black hole with a non-de Sitter core is obtained.

In order to show it, let us study other possible identifications different to the spacelike one previously considered. One possibility is to identify the sections corresponding to $r=r_{-}$and see if the rest of the hypothesis can be left untouched. This is indeed the case, as Fig. (3) shows.

In Fig. (3), the shaded region represents the chronological future of $p$, the boundary of this region being equal to the future horismo of $p$. Therefore, after this null identification, the spacetime remains future causally simple. In addition, Fig. (3) reveals that it is future null geodesically complete but not simply connected since the regions between $r=r_{-}$and $r=0$ are excluded from it.

The topology of the achronal slices constructed by means of the null identification along $r_{-}$can be understood as follows. 


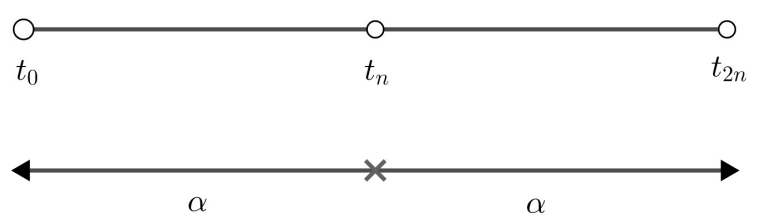

FIG. 4. Line segment for null identification study.

First, the key point is to show that the line segment along the Cauchy horizon can be deformed to a circle. With the notation established in Fig. (4), we will say that two points $t_{i}, t_{j}$ are equivalent if $t_{j}=t_{i}+n \alpha, i \leq j$ and $n \in \mathbb{Z}$. Let us see if this relation defines an equivalence relation.

1. First let's see that $t_{i}$ is related to itself:

$$
t_{i}-t_{i}=0 \equiv 0 \times \alpha, \quad 0 \in \mathbb{Z} \Rightarrow t_{i} \sim t_{i}
$$

2. Then, if

$$
\begin{aligned}
& t_{i} \sim t_{j} \Rightarrow t_{j}-t_{i}=n \alpha \rightarrow t_{i}-t_{j}=-n \alpha \\
& (-n) \in \mathbb{Z} \Rightarrow t_{j} \sim t_{i}
\end{aligned}
$$

\section{Finally, if}

$$
\begin{aligned}
& t_{i} \sim t_{j}, t_{j} \sim t_{c} \Rightarrow t_{j}-t_{i}=n \alpha, t_{c}-t_{j}=n^{\prime} \alpha, \\
& t_{c}-t_{i}=t_{c}-\left(t_{j}-n \alpha\right)=\left(n^{\prime}+n\right) \alpha, \\
& \left(n^{\prime}+n\right) \in \mathbb{Z} \Rightarrow t_{c} \sim t_{i} .
\end{aligned}
$$

Then, the relation defined above is an equivalence relation. In this way we can see that $t_{0} \sim t_{n}, t_{1} \sim t_{n+1}$, $t_{2} \sim t_{n+2}, t_{0} \sim t_{2 n}$ and, as Fig. (5) shows, the line segment together with this identification corresponds to a circle without a point. Therefore, the topology of the slices taken along $r=r_{-}$is $\left[S^{1}-\left\{t_{0}\right\}\right] \times S^{2}$.

Finally, as this slice is non-compact, we need to make an Alexandroff extension to compactify it by adding a point at the $i^{-}$infinity. In this way, the obtained topology is that of $S^{1} \times S^{2}$. We remark that the addition of this point in order to makes the compactification does not alter any property of the slice, in particular the property of being edgeless and achronal.

We can also make another identification that is future causally simple and that satisfies Borde's theorem but in which the space is non-orientable. For this identification, the sections corresponding to $r=r_{-}$will be identified in the way shown in Fig. (6), so it can be demonstrated (in the same way as for the orientable null identification) that the topology of the slices shown by dotted lines (orange and violet) is $S^{1} \times S^{2}$ but with the particularity that the outgoing null rays reach the null infinite of the ingoing rays and vice versa. Therefore, the $S^{2}$ bundle is placed in a non-orientable way on $S^{1}$ and the topology
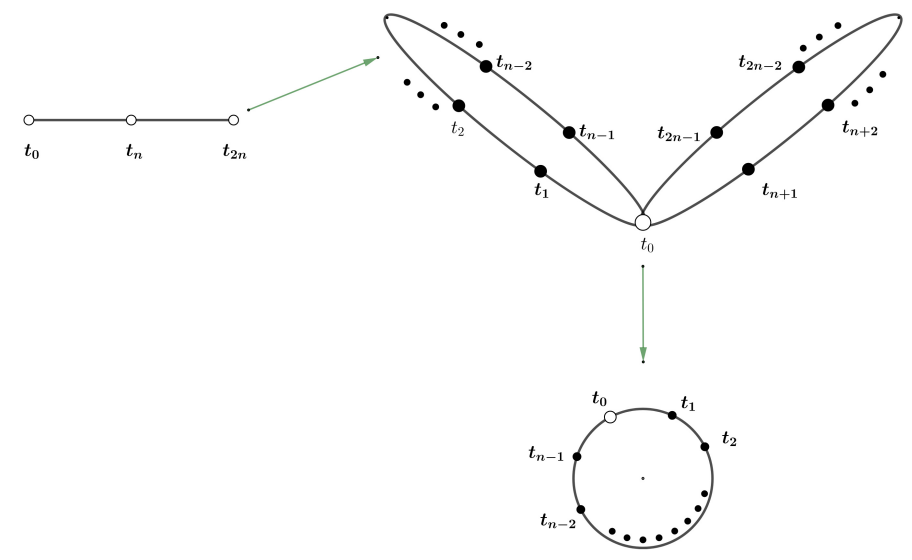

FIG. 5. Diagram showing that lightlike identification along a line segment gives place to a circle.

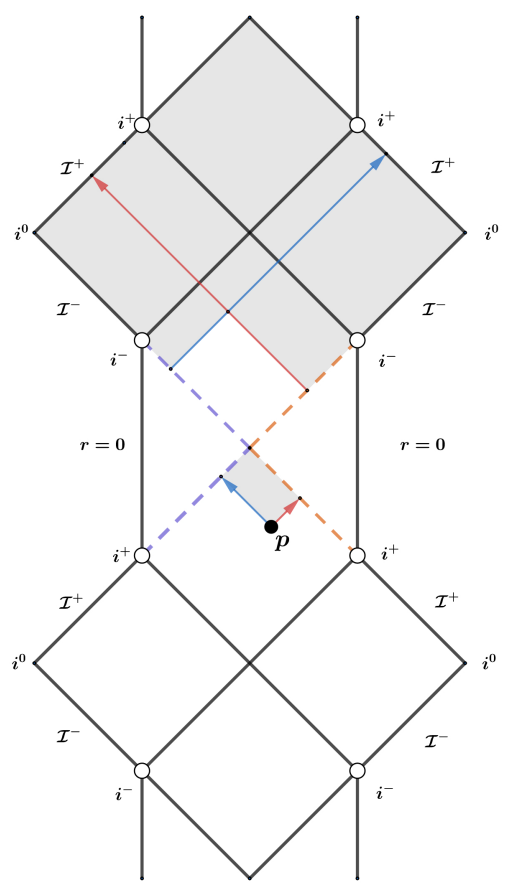

FIG. 6. Carter-Penrose diagram for non-orientable null identification along the Cauchy horizon.

of the null slices corresponding to this identification is $S^{2} \tilde{\times} S^{1}$ (the non-orientable $S^{2}$ bundle over $S^{1}$ ).

All these results can be summarized in the following

Theorem. Let $\mathcal{M}$ be a spherically symmetric and static spacetime such that

1. $\mathcal{M}$ contains an eventually future-trapped surface $\mathcal{T}$.

2. The Ricci tensor, $R_{\mu \nu}$, obeys $R_{\mu \nu} n^{\mu} n^{\nu} \geq 0$ for all null vectors $n^{\mu} \in \mathcal{M}$.

3. $\mathcal{M}$ is null-geodesically complete to the future.

4. $\mathcal{M}$ is future causally simple, i.e., $E^{+}(X)=\dot{I}^{+}(X)$, where $X$ is any achronal compact subset of $\mathcal{M}$. 
Then there is a compact slice to the causal future of $\mathcal{T}$ whose topology is

1. $S^{3}$ if $R_{\mu \nu} n^{\mu} n^{\nu}=0$ at $r=0$

2. $S^{1} \times S^{2}$ if $R_{\mu \nu} n^{\mu} n^{\nu}>0$ for all $p \in \mathcal{M}$ and $\mathcal{M}$ is orientable

3. $S^{1} \tilde{\times} S^{2}$ if $R_{\mu \nu} n^{\mu} n^{\nu}>0 p \in \mathcal{M}$ and $\mathcal{M}$ is not orientable

Even more, regarding connectivity, we observe that in cases 2 and 3 of the previous theorem $\mathcal{M}$ is not simply connected. Therefore, assuming connectivity (which is a physically desirable property for a spacetime) for a spherically symmetric and static spacetime, together with hypothesis 1-4 of Borde's theorem, we are led to a regular black hole with a de Sitter core $\left(S^{3}\right.$ topology for the compact achronal slice) as the only possibility. In this sense, we think that there is no physical reason to look for new regular black hole solutions with the exotic topologies here studied since they can not incorporate the connectivity of $\mathcal{M}$.

As a summary, our main findings are presented in Table (I).

\begin{tabular}{|c|c|c|}
\hline Slice & Compactification & Topology \\
\hline 0 to $r_{-}$ & spacelike & $S^{3}$ \\
$r_{-}$to $r_{+}$ & spacelike & $R \times S^{2}$ \\
$r_{+}$to $\infty$ & spacelike & $R \times S^{2}$ \\
0 to $r_{-}$ & orientable lightlike & $S^{1} \times S^{2}$ \\
$r_{-}$to $r_{+}$ & orientable lightlike & $R \times S^{2}$ \\
$r_{+}$to $\infty$ & orientable lightlike & $R \times S^{2}$ \\
0 to $r_{-}$ & non-orientable lightlike & $S^{1} \tilde{\times} S^{2}$ \\
\hline
\end{tabular}

TABLE I. Topologies for different achronal slices found in spherically symmetric regular black hole spacetimes.

Interestingly, it is worth mentioning that, in previous works (see, for example, Ref. ${ }^{11}$ ), several regular black hole solutions were obtained with the only requirement that the mass distribution had to be regular at the center and the solution should be either asymptotically Reissner-Nordström or satisfies the weak energy condition (or both of them). In all the cases, the core of the solution had a de Sitter center but nothing was commented about Borde's theorem conditions. In this sense, our result allows to classify previously obtained regular black hole solutions in a simple manner such as, for example, those presented in Refs. ${ }^{8,11,24,25}$, which lie into the first case of our theorem.

\section{QUANTIFICATION OF TOPOLOGY CHANGE IN REGULAR BLACK HOLE SPACETIMES}

From Borde's theorem we have seen that, for regular black hole spacetimes, the topology of certain achronal hypersurfaces changes in regions near the core (or near the Cauchy horizon in case of lightlike compactification). In this section we are interested in defining quantities related to physical variables of the system that allow us to determine the topology of the relevant slices in order to connect physical quantities with topological changes. In order to simplify the discussion, and having into account that we are interested in simply connected spacetimes, only the spacelike regularization will be considered.

Regarding this point we would like to mention the work of Refs. ${ }^{26,27}$, where the authors attributed the changes in topology to a change in the sign of the scalar curvature. In the case they studied, they used a type- $I$ energy-momentum tensor satisfying $T_{0}^{0}=T_{r}^{r}$. In this case, $R \propto\left(\rho-p_{\perp}\right)$ and it can be seen that $R<0$ when the dominant energy condition is violated. Therefore, in this case, the topology change is attributed to the violation of the dominant energy condition. However, as some regular black hole solutions satisfying the dominant energy condition are known ${ }^{6,7}$, we are not completely sure about the kind of topology change the authors of these works refer to. In addition, this change of sign is difficult to be interpreted as a global (topological) feature, unless it completely modifies a globally defined magnitude, which seems not to be the case presented in Refs. ${ }^{26,27}$ because the local character of the Ricci scalar, $R$.

As an alternative to the previous proposal, the authors of Ref. ${ }^{28}$ studied the energy conditions in regular black hole spacetimes with several horizons, managing to relate the sign of the Tolman mass, $m_{T}\left(r_{a}, r_{b}\right)$, defined between the radial coordinates $\left(r_{a}, r_{b}\right)$ with the violation of strong energy condition ${ }^{39}$. In particular, it can be seen that $m_{T}\left(r_{a}, r_{b}\right)$ is negative in the region $0 \leq r<r_{-}$(in this region we have compact achronal hypersurfaces) and, in the regions $r_{-}<r<r_{+}$and $r_{+}<r<\infty, m_{T}\left(r_{a}, r_{b}\right)$ remains positive.

Let's detail this idea. Following ${ }^{28}$ we will use a spherically symmetric line element

$$
d s^{2}=-f(r) d t^{2}+\frac{d r^{2}}{f(r)}+r^{2} d \Omega^{2}
$$

Using Eq. (4) we arrive at

$$
-R_{0}^{0} r^{2}=\frac{1}{2} \frac{d}{d r}\left(r^{2} \frac{d f}{d r}\right)
$$

whose integration leads to

$$
2 m_{T}\left(r_{a}, r_{b}\right)=\left.\left(r^{2} \frac{d f}{d r}\right)\right|_{r_{a}} ^{r_{b}}
$$

where by definition ${ }^{29}$

$$
m_{T}\left(r_{a}, r_{b}\right)=4 \pi \int_{r_{a}}^{r_{b}} d r r^{2}\left(T_{k}^{k}-T_{0}^{0}\right) .
$$


is the Tolman mass.

If we evaluate this mass for the Reissner-Nordström black hole $\left(f=1-\frac{2 M}{r}+\frac{Q^{2}}{r^{2}}\right)$ in the region $\epsilon<r<r_{-}$, we have the following:

$$
\left.\left(r^{2} \frac{d f}{d r}\right)\right|_{\epsilon} ^{r_{-}}=2 Q^{2}\left(\frac{1}{\epsilon}-\frac{1}{r_{-}}\right)
$$

In the case when $\epsilon$ tends to zero, we see that Eq. (8) is always positive. For the $r_{-}<r<r_{+}$and $r_{+}<r<\infty$ intervals the following results are obtained respectively

$$
\left.\left(r^{2} \frac{d f}{d r}\right)\right|_{r_{-}} ^{r_{+}}=2 Q^{2}\left(\frac{1}{r_{-}}-\frac{1}{r_{+}}\right)
$$

and

$$
\left.\left(r^{2} \frac{d f}{d r}\right)\right|_{r_{+}} ^{\infty}=2 \frac{Q^{2}}{r_{+}}
$$

Therefore, Tolman's mass in the studied regions for the singular case meet the following

- $m_{T}\left(r_{+}, \infty\right)>0$.

- $m_{T}\left(r_{-}, r_{+}\right)>0$.

- $m_{T}\left(0, r_{-}\right)>0$.

Now let's analyze the regular cases studied in this work. The right side of Eq. (6) can be rewritten as follows

$$
\left.\left(r^{2} \frac{d f}{d r}\right)\right|_{r_{a}} ^{r_{b}}=\left.r_{b}^{2} \frac{d f}{d r}\right|_{r_{b}}-\left.r_{a}^{2} \frac{d f}{d r}\right|_{r_{a}} .
$$

When $r \rightarrow \infty$ the metric is asymptotically flat and can be written as

$$
f \approx 1-\frac{2 M}{r}
$$

from the above it is obtained that $\frac{d f}{d r}=\frac{2 M}{r^{2}}$. Also, it can be seen in Fig. (1) that the spatial coordinate $r$ behaves like a temporal coordinate in region $r_{-}<r<r_{+}$and vice versa, in the metric this manifests like a sign change of $f$, being positive for $r>r_{+}$and negative when $r_{-}<r<r_{+}$, from this we get that $\left.\frac{d f}{d r}\right|_{r_{+}}>0$. Then, in region $0<r<$ $r_{-}$, the function $f$ changes sign, becoming positive again, reason why the usual behavior of the coordinates $r$ and $t$ is recovered, in this way we have that $\left.\frac{d f}{d r}\right|_{r_{-}}<0$.
So, from what was obtained previously, equation (11) can now be evaluated in the interest regions. In interval $\left(r_{+}, \infty\right)$ we have

$$
\left.\left(r^{2} \frac{d f}{d r}\right)\right|_{r_{+}} ^{\infty}=2 M-r_{+}^{2} f^{\prime}\left(r_{+}\right)=2 M-2 r_{+}^{2} \kappa,
$$

where $\kappa$ is the surface gravity of the regular black hole. So the Tolman mass in this interval is $m_{T}\left(r_{+}, \infty\right)=$ $M-r_{+}^{2} \kappa$, the right side of this equality will be positive if the strong energy condition or the dominant energy condition $^{40}$ is satisfied in this region ${ }^{30}$, then we will assume cases where one of these energy conditions is met.

For $\left(r_{-}, r_{+}\right)$interval, is obtained that

$$
\left.\left(r^{2} \frac{d f}{d r}\right)\right|_{r_{-}} ^{r_{+}}=\left.r_{+}^{2} \frac{d f}{d r}\right|_{r_{+}}-\left.r_{-}^{2} \frac{d f}{d r}\right|_{r_{-}},
$$

since the second term on the right is negative, the Tolman mass for that region is positive. Finally, in $\left(0, r_{-}\right)$interval we have

$$
\left.\left(r^{2} \frac{d f}{d r}\right)\right|_{0} ^{r_{-}}=\left.r_{+}^{2} \frac{d f}{d r}\right|_{r_{-}},
$$

resulting in a negative Tolman mass for this region.

Can be noticed that the Tolman's mass sign for the $\left(0, r_{-}\right)$interval differs between the regular and singular cases, and it is precisely in this region that the topology change occurs for the spatial slices of the regular black holes analyzed in this work. This fact makes us to think that there could be a relation between topology change and the sign of the Tolman mass. In adition, we support this conjecture on the fact that the topology of null infinity changes from $R \times S^{2}$ when the Bondi mass vanishes to $S^{3}$ for a non vanishing Bondi mass ${ }^{31,32}$. Therefore, as the Bondi mass roughly corresponds to the null version of the Tolman mass, we are led to conjecture that $m_{T}$ could be related to the topology change of the spacelike slices.

Finally, trying to find a different strategy in order to physically quantify the topology change, a couple of comments are in order. First, in this work we are considering spherically symmetric solutions. Importantly, they are of Petrov type D at any point except at $r=0$, which they turn into O-type (conformally flat, which is in agreement with the presence of a de Sitter core). Therefore, some phase transition such as that explored in Refs. ${ }^{33,34}$ could be valuable in order to shed some light on the mechanism of topology change. Second, we think that Morse Theory should be considered as a serious candidate in order to explore this issues. Although some previous works on this direction can be found in the literature ${ }^{35,36}$, we have not found applications to the case of regular black holes. Even more, due to the type of geometries we are considering, the only non vanishing Newman-Penrose scalars are $\Psi_{2}$ and $\Lambda^{37}$. Therefore, we think that it would be interesting to look for physically-relevant Morse functions 
defined only in terms of $\Psi_{2}$ and $\Lambda$ in order to link them with topological changes due to regularization.

\section{CONCLUSIONS}

In this work we have studied all the possibilities that Borde's theorem provide in order to obtain regular black hole solutions within spherical symmetry. We have shown that both spacelike and lightlike compactifications give place to regular black hole spacetimes with a de Sitter $\left(S^{3}\right), S^{1} \times S^{2}$ (orientable) or $S^{1} \tilde{\times} S^{2}$ (non-orientable) compact achronal and edgeless hypersurfaces. Interestingly, if simply connectedness is imposed as a reasonable property for the spacetime, only the inflationary de Sitter core survives. Finally, we have discussed possible ways of quantitatively detecting topology change is these spacetimes, trying to open new routes for future research.

\section{ACKNOWLEDGEMENTS}

P. B. is funded by the Beatriz Galindo contract BEAGAL 18/00207 (Spain).
* ga.melgarejo@uniandes.edu.co

† econtreras@usfq.edu.ec

¥ pedro.bargueno@ua.es

1 R. Penrose. Gravitational Collapse and Space-Time Singularities. Physical Review Letters, 14:57-59, 1965. DOI:10.1103/PhysRevLett.14.57.

2 J. Bardeen. Proceedings of GR5, Tiflis, USSR, 1968.

3 A. D. Sakharov. The initial stage of an expanding universe and the appearance of a nonuniform distribution of matter. J. Exptl. Theoret. Phys. (U.S.S.R.), 22(1):345-358, 1996.

${ }^{4}$ E. B. Gliner. Algebraic properties of the energymomentum tensor and vacuum-like states of matter. $J$. Exptl. Theoret. Phys, 22(2):42-548, 1966.

${ }^{5}$ I. G. Dymnikova. Vacuum nonsingular black hole, General Relativity and Gravitation, 1992. DOI: 10.1007/BF00760226.

${ }^{6}$ I. G. Dymnikova. The cosmological term as a source of mass, Classical and Quantum Gravity, 1992. DOI: 10.1088/0264-9381/19/4/306.

7 I. G. Dymnikova. Spherically symmetric space-time with regular de Sitter center. International Journal of Modern Physics D, 12, 2003. DOI: 10.1142/S021827180300358X.

8 I. G. Dymnikova. Regular electrically charged vacuum structures with de Sitter centre in nonlinear electrodynamics coupled to general relativity. Classical and Quantum Gravity, 2004. DOI: 10.1088/0264-9381/21/18/009.

9 E. Ayón-Beato and A. García. The Bardeen model as a nonlinear magnetic monopole. Physics Letters B, 493:149152, 2000. pages $=149-152$, volume $=493$, DOI: 10.1016/S0370-2693(00)01125-4.

10 J. Lemos and V. Zanchin. Regular black holes: Electrically charged solutions, Reissner-Nordström outside a de Sitter core. Physical Review D - Particles, Fields, Gravitation and Cosmology, 83(12), 6 2011. DOI: 10.1103/PhysRevD.83.124005.

${ }^{11}$ L. Balart and Elias C. Vagenas. Regular black holes with a nonlinear electrodynamics source. Physical Review D Particles, Fields, Gravitation and Cosmology, 90(12), 12 2014. DOI: 10.1103/PhysRevD.90.124045.

12 N. Morales-Durán, A. F. Vargas, P. Hoyos-Restrepo, and P. Bargueño. Simple regular black hole with logarithmic entropy correction. European Physical Journal C, 76(10), 10 2016. DOI: 10.1140/epjc/s10052-016-4417-x.

13 J. Aftergood and A. Debenedictis. Matter conditions for regular black holes in $\mathrm{f}(\mathrm{T})$ gravity. Physical Review D -
Particles, Fields, Gravitation and Cosmology, 90(12), 12 2014. DOI: 10.1103/PhysRevD.90.124006.

14 M. E. Rodrigues, E. L. B. Junior, G, T. Marques, and V. T. Zanchin. Regular black holes in $\mathrm{f}(\mathrm{R})$ gravity coupled to nonlinear electrodynamics. Physical Review D, 94(2), 7 2016. DOI: 10.1103/PhysRevD.94.024062.

15 E. Contreras, Á. Rincón, B. Koch, and P. Bargueño. A regular scale-dependent black hole solution. International Journal of Modern Physics D, 27(3), 2 2018. DOI: 10.1142/S0218271818500323.

16 A. Simpson and M. Visser. Regular Black Holes with Asymptotically Minkowski Cores. Universe, 6(1):8, 12 2019. DOI: 10.3390/universe6010008.

17 A. Borde. Regular black holes and topology change. Physical Review D - Particles, Fields, Gravitation and Cosmology, 1997. DOI: 10.1103/PhysRevD.55.7615.

18 R. Carballo-Rubio, F. Di Filippo, S. Liberati, and M. Visser. Opening the Pandora's box at the core of black holes, 8 2019. arXiv: 1908.03261.

19 R. Carballo-Rubio, F. Di Filippo, S. Liberati, and M. Visser. Geodesically complete black holes, 11 2019. arXiv: 1911.11200.

20 Stephen Hawking and Roger Penrose. The nature of space and time. Princeton University Press, 2010.

21 Robert. M. Wald. General relativity. University of Chicago Pres, Chicago, IL, 1984.

22 S. Ansoldi. Spherical black holes with regular center: a review of existing models including a recent realization with Gaussian sources. Delivered at the conference "Dynamics and Thermodynamics of Blackholes and Naked Singularities", Department of Mathematics of the Politecnico, May 10-12 2007, Milano, Italy, 2008. arXiv: 0802.0330v1.

${ }^{23}$ L. Balart and E. C. Vagenas. Regular black hole metrics and the weak energy condition. Physics Letters, Section B: Nuclear, Elementary Particle and High-Energy Physics, 2014. DOI: 10.1016/j.physletb.2014.01.024.

24 E. Ayón-Beato and A. García. Regular Black Hole in General Relativity Coupled to Nonlinear Electrodynamics. Physical Review Letters, 80(23):5056-5059, 1998. DOi: 10.1103/PhysRevLett.80.5056.

25 E. Ayón-Beato and A. García. Four-parametric regular black hole solution. General Relativity and Gravitation, 37(4):635-641, 4 2005. DOI: 10.1007/s10714-005-0050-y.

26 I. G. Dymnikova and E. Galaktionov. Stability of a vacuum non-singular black hole. Classical and Quantum 
Gravity, 22(12):2331-2357, 6 2005. DOi: 10.1088/0264$9381 / 22 / 12 / 003$.

27 M. Azreg-Aïnou. Smoothed one-core and core-multi-shell regular black holes. European Physical Journal C, 78(6), 6 2018. DOI: 10.1140/epjc/s10052-018-5966-y.

${ }^{28}$ O. B. Zaslavskii. Regular black holes and energy conditions. Physics Letters, Section B: Nuclear, Elementary Particle and High-Energy Physics, 688(4-5):278-280, 5 2010.DOI: 10.1016/j.physletb.2010.04.031.

29 R.C. Tolman. Relativity, Thermodynamics, and Cosmology. New York: Dover Publications, 1987.

30 Markus Heusler. A mass bound for spherically symmetric black hole spacetimes. Class. Quantum Grav 12 (1995), 779-789. DOI: 10.1088/0264-9381/12/3/015.

31 A. Magnon. Global techniques, dual mass, and causality violation. Journal of Mathematical Physics, 27(4):1066-1073, 1986. DOI: $10.1063 / 1.527149$.

32 S. Ramaswamy and A. Sen. Dual-mass in general relativity. Journal of Mathematical Physics, 22(11):2612-2619, 11 1981. DOI: $10.1063 / 1.524839$.

33 A. M. Baranov. Gravitational fields of lightons and helixons in General Relativity. arXiv preprint, 2011. arXiv: $1105.6327 \mathrm{v} 1$.

34 A. M. Baranov. The catastrophe theory, Petrov's algebraic classification and gravitational phase transitions. Gravitation and Cosmology, 1782):170-172, 4 2011. DOI:
10.1134/S0202289311020058

35 H. F. Dowker, R. S. Garcia, and S. Surya. Morse index and causal continuity. A criterion for topology change in quantum gravity. Class. Quantum Grav, 17:697-712, 2000. DOI: $10.1088 / 0264-9381 / 17 / 3 / 308$.

36 A. Borde, F. Dowker, R. S. Garcia, R. D. Sorkin, and S. Surya. Causal continuity in degenerate spacetimes. Class. Quantum Grav, 16:3457-3481, 1999. DOI: 10.1088/02649381/16/11/303.

37 R. Penrose anmd W. Rindler. Spinors and Space-Time: Volume 1, Two-Spinor Calculus and Relativistic Fields. Cambridge University Press, 1984.

38 As commented by Lemos ${ }^{10}$, a useful way to classify this regular spacetimes is: (i) no junction (the solution is continuous throughout the whole spacetime), (ii) two simple regions (the solution has boundary surfaces joining the two regions), (iii) two regions (the solution has a surface layer joining the two regions). However, in this work we are mainly interested in the first class.

39 The strong energy condition states that $R_{\mu \nu} \xi^{\mu} \xi^{\nu} \geq 0$, for all time-like vectors $\xi^{\mu}$.

40 The dominant energy condition states that for all time-like vectors $\xi^{\mu}$ it must be fulfilled that $G_{\mu \nu} \xi^{\mu} \xi^{\nu} \geq 0$ and $G^{\mu}{ }_{\nu} \xi^{\nu}$ must be causal, where $G^{\mu}{ }_{\nu}$ is Einstein's tensor. 